OPEN ACCESS

Edited by:

Marko Djordjevic,

University of Belgrade, Serbia

Reviewed by:

Katarina Zeliic,

University of Belgrade, Serbia

Songjie Shen,

Peking Union Medical College

Hospital (CAMS), China

*Correspondence:

Lin L

fcclil6@zzu.edu.cn

Specialty section:

This article was submitted to

Computational Genomics,

a section of the journal

Frontiers in Genetics

Received: 07 June 2021

Accepted: 09 July 2021

Published: 02 August 2021

Citation:

Wang N, Gu Y, Chi J, Liu X, Xiong $Y$, Zhong $C$, Wang $F$, Wang $X$ and Li L (2021) Screening of DNA

Damage Repair Genes Involved in the Prognosis of Triple-Negative Breast Cancer Patients Based on

Bioinformatics.

Front. Genet. 12:721873 doi: 10.3389/fgene.2021.721873

\section{Screening of DNA Damage Repair Genes Involved in the Prognosis of Triple-Negative Breast Cancer Patients Based on Bioinformatics}

\author{
Nan Wang ${ }^{1}$, Yuanting Gu${ }^{1}$, Jiangrui Chi' ${ }^{1}$, Xinwei Liu' ${ }^{1}$, Youyi Xiong ${ }^{1}$, Chaochao Zhong ${ }^{2}$, \\ Fang Wang ${ }^{1}$, Xinxing Wang ${ }^{1}$ and Lin $\mathrm{Li}^{1 *}$ \\ ${ }^{1}$ Department of Breast Surgery, The First Affiliated Hospital of Zhengzhou University, Zhengzhou, China, ${ }^{2}$ Department \\ of Plastic Surgery, The First Affiliated Hospital of Zhengzhou University, Zhengzhou, China
}

Background: Triple-negative breast cancer (TNBC) is a special subtype of breast cancer with poor prognosis. DNA damage response (DDR) is one of the hallmarks of this cancer. However, the association of DDR genes with the prognosis of TNBC is still unclear.

Methods: We identified differentially expressed genes (DEGs) between normal and TNBC samples from The Cancer Genome Atlas (TCGA). DDR genes were obtained from the Molecular Signatures Database through six DDR gene sets. After the expression of six differential genes were verified by quantitative real-time polymerase chain reaction (qRT-PCR), we then overlapped the DEGs with DDR genes. Based on univariate and LASSO Cox regression analyses, a prognostic model was constructed to predict overall survival (OS). Kaplan-Meier analysis and receiver operating characteristic curve were used to assess the performance of the prognostic model. Cox regression analysis was applied to identify independent prognostic factors in TNBC. The Human Protein Atlas was used to study the immunohistochemical data of six DEGs. The prognostic model was validated using an independent dataset. Gene Ontology and the Kyoto Encyclopedia of Genes and Genomes analysis were performed by using gene set enrichment analysis (GSEA). Single-sample gene set enrichment analysis was employed to estimate immune cells related to this prognostic model. Finally, we constructed a transcriptional factor (TF) network and a competing endogenous RNA regulatory network.

Results: Twenty-three differentially expressed DDR genes were detected between TNBC and normal samples. The six-gene prognostic model we developed was shown to be related to OS in TNBC using univariate and LASSO Cox regression analyses. All the six DEGs were identified as significantly up-regulated in the tumor samples compared to the normal samples in qRT-PCR. The GSEA analysis indicated that the 
genes in the high-risk group were mainly correlated with leukocyte migration, cytokine interaction, oxidative phosphorylation, autoimmune diseases, and coagulation cascade. The mutation data revealed the mutated genes were different. The gene-TF regulatory network showed that Replication Factor $\mathrm{C}$ subunit 4 occupied the dominant position.

Conclusion: We identified six gene markers related to DDR, which can predict prognosis and serve as an independent biomarker for TNBC patients.

Keywords: DNA damage response, prognostic model, bioinformatics and computational biology, triple-negative breast cancer, biomarker discovery

\section{INTRODUCTION}

Breast cancer is the most common malignancy in women. The International Agency for Research on Cancer of the World Health Organization reported that the number of new cases of breast cancer reached 2.26 million in 2020, and breast cancer has become the most prevalent malignant tumor in the world (Sung et al., 2021). Breast cancer is a biologically and clinically heterogeneous disease with several recognized tissue and molecular subtypes with different etiologies, risk factors, treatment responses, and prognoses (Allemani et al., 2018; Mavaddat et al., 2019; Pashayan et al., 2020). Triple-negative breast cancer (TNBC) is defined as a type of breast cancer with negative expression of estrogen (ER), progesterone (PR), and human epidermal growth factor receptor-2 (HER2; Bianchini et al., 2016). Compared with other breast cancer subtypes, TNBC is highly aggressive and has a high rate of early recurrence. Patients with TNBC usually relapse within 5 years after surgery, and the overall prognosis is very poor (Emens, 2018). Due to the lack of ER, PR, and HER2 expression in TNBC tumors, few therapies targeting specific molecular targets have been able to significantly improve the prognosis of patients with TNBC disease, and chemotherapy remains the standard of treatment of TNBC (Bianchini et al., 2016; Lee et al., 2020). Although many patients with early-stage TNBC disease are cured by chemotherapy, the overall median survival with the current treatment regimen is 13-18 months among those who develop metastatic disease (André and Zielinski, 2012). Therefore, in order to improve the prognosis and curative effect of TNBC patients, it is urgent to obtain new and effective biomarkers.

\footnotetext{
Abbreviations: AUC, under the ROC curve; BC, breast cancer; BER, base excision repair; ceRNAs, competing endogenous RNAs; CPF, checkpoint factor; DDR, DNA damage response; DEGs, differentially expressed genes; DFS, disease-free survival; EXO1, Exonuclease 1; FA, Fanconi's anemia; GEO, Gene Expression Omnibus; GO, Gene Ontology; GSEA, gene set enrichment analysis; HER2, human epidermal growth factor receptor-2; HRR, homologous recombination repair; IARC, International Agency for Research on Cancer; KEGG, Kyoto Encyclopedia of Genes and Genomes; MDSCs, myeloid-derived suppressor cells; MFE, minimal folding free energy; MMR, Mismatch repair; MSigDB, Molecular Signatures Database; NER, nucleotide excision repair; NHEJ, non-homologous end ligation; NPM1, nucleic acid-binding domain of nucleophosmin; OS, overall survival; PAPRi, poly-ADP-ribose polymerase inhibitor; PR, progesterone; RFC, Replication Factor C; RMI2, RecQ-mediated genome instability protein 2; ROC, receiver operating characteristic; SAC, spindle assembly point; SNP, single-nucleotide polymorphism; ssGSEA, single-sample gene set enrichment analysis; TCGA, the Cancer Genome Atlas; TF, transcriptional factor; TLS, Translesion Synthesis; TMB, tumor mutation burden; TNBC, triple-negative breast cancer.
}

DNA damage response (DDR; Cancer Genome Atlas Research Network, 2017. Electronic address and Cancer Genome Atlas Research) pathways are an important mechanism to correct and repair DNA damage, which can inhibit cell aging, apoptosis, and carcinogenesis in time and ensure normal life activities (Roos et al., 2016). DDR consists of eight pathways: (1) mismatch repair (MMR), (2) base excision repair (BER), (3) nucleotide excision repair (NER), (4) homologous recombination repair (HRR), (5) non-homologous end ligation (NHEJ), (6) checkpoint factor (CPF), (7) Fanconi's anemia (FA), and (8) variable DNA synthesis (TLS). The interaction of these pathways can repair DNA damage accurately and timely, prevent gene distortion, and ensure the integrity of the genome (Scarbrough et al., 2016). Recent studies have shown that increasing DNA damage and reducing the DNA repair ability of cancer cells lead to genome distortion of cancer cells, but that distinguishing these cells from normal cells can improve the effectiveness of cancer treatment (Lawrence et al., 2014). DDR genes can broaden therapy options for cancer patients by cancerdriving effects and significance in clinical and translational medicine (Hu and Guo, 2020). For example, DDR alterations are independently associated with the therapeutic response to PD-1/PD-L1 inhibitors and are positively correlated with a higher tumor mutation burden (Turner et al., 2004; Cerrato et al., 2016). Poly-ADP-ribose polymerase inhibitor therapy had a better effect on cancer patients with BRCA1/2 mutations (Faraoni and Graziani, 2018; Teo et al., 2018). Moreover, many studies have demonstrated that tumors with deleterious DDR mutations are more sensitive to platinum-based therapy (Tutt et al., 2018). Therefore, DDR genes are very important to the prognosis of patients, but there has yet to be a systematic study of DDR genes in TNBC.

In this study, we downloaded the sequencing data and DDR datasets of TNBC patients from The Cancer Genome Atlas (TCGA) and the Gene Expression Omnibus (GEO) databases and performed bioinformatics analysis on them to comprehensively evaluate whether the expression level of DDR-related genes can predict the prognosis of TNBC patients. The aim of our study was to identify new potential prognostic markers for TNBC and establish new prognostic models to assist in the formulation of diagnosis and treatment strategies. In addition, we stratified the risk of TNBC patients by establishing a prognostic model, and then performed more specific treatments on the patients according to the results of the different risk assessments, so as to avoid unnecessary active treatments for the patients. 


\section{MATERIALS AND METHODS}

\section{Clinical Sample Acquisition}

Tumor tissue samples and adjacent normal breast tissue samples from 10 TNBC patients were obtained from the sample library of The First Affiliated Hospital of Zhengzhou University, Henan, China. All the patients had received surgery in The First Affiliated Hospital of Zhengzhou University and had not received any anti-cancer treatment before surgery. Tissue specimens were collected within $30 \mathrm{~min}$ after surgery and quickly frozen in liquid nitrogen. Postoperative monitoring and treatment continued in accordance with the relevant consensus guidelines. Tumors were graded according to the WHO grading system. Each patient's written informed consent to donate the sample to the sample bank was required before any sample was collected. Our research was approved by the Ethics Committee of The First Affiliated Hospital of Zhengzhou University and was conducted in accordance with the principles of Declaration of Helsinki. The age and clinical conditions of each patient, such as immunohistochemistry, are shown in Supplementary Table 1.

\section{Data Collection and Differential Expression Analysis}

The gene expression data and clinical information of TNBC patients were acquired from the TCGA database ${ }^{1}$ and GEO database $^{2}$. We collected 99 normal and 113 TNBC samples from the TCGA database. Differentially expressed genes (DEGs) were selected using the "limma" package ( $\mid \log _{2}$ (fold change) $\mid>1$, $P<0.05)$. We obtained six DDR gene sets that encompassed several DDRs pathways, including MMR, BER, CPFs, NER, HRR, FA, and NHEJ, from Molecular Signatures Database (MSigDB) ${ }^{3}$ and 57 duplicate DDRs were removed. Finally, we collected 154 DDR genes and intersected them with DEGs (DE-DDRs).

\section{Survival Analysis}

From a total of 113 TNBC samples, after excluding a TNBC sample lacking survival data, we used 112 TNBC samples to construct a prognostic model. Univariate and LASSO Cox analyses were used to identify prognostic genes that were significantly associated with OS (overall survival). Univariate Cox analysis was used to initially identify the potential prognostic genes $(P<0.2$; Kang et al., 2013). Next, we used the R package "glmnet" for the LASSO Cox regression analysis to construct the prognostic model for TNBC patients. The risk score was calculated according to the standardized expression level of each gene and its corresponding regression coefficient. The following formula was used: Risk score = $\left(\right.$ Coefficient $_{\text {mRNA } 1} \times$ Expression $\left._{\text {mRNA } 1}\right)+\left(\right.$ Coefficient $_{\text {mRNA2 }} \times$ Expression $\left._{\text {mRNA2 }}\right)+\cdots+\left(\right.$ Coefficient $_{m R N A n} \times$ Expression mRNAn). Setting the median risk score of the TCGA database as the cut-off value, 112 TNBC samples were divided into lowor high-risk groups. The R package "survival" was performed

\footnotetext{
${ }^{1}$ https://portal.gdc.cancer.gov/cart

${ }^{2}$ https://www.ncbi.nlm.nih.gov/gds

${ }^{3}$ http://www.gsea-msigdb.org/gsea/msigdb/
}

to generate the K-M survival curve, and the $\mathrm{R}$ package "survivalROC" was used to generate time-dependent receiver operating characteristic (ROC) curves to evaluate the predictive power of the prognostic model. The GSE58812 dataset, which contained 107 TNBC samples, was used for validation of the above results. Univariate and multivariate Cox regression analyses were performed to analyze the independent prognosis of the six-gene prognostic model. All independent prognostic factors were used to construct a nomogram to predict the survival of TNBC patients at 3 and 5 years. In addition, survival analysis of six prognostic genes was performed separately using the TCGA database.

\section{RNA Extraction and Quantitative Real-Time Polymerase Chain Reaction}

Total RNA was isolated from 10 paired tissues using Nuclezol LS RNA Isolation Reagent (ABP Biosciences Inc) according to the instructions. Then, we quantified the concentration and purity of the RNA solution using an Ultraviolet spectrophotometer (Life Real). Briefly, the extracted RNA was reverse-transcribed to cDNA using the SureScript-First-strand-cDNA-synthesis-kit (GeneCopoeia) prior to quantitative real-time polymerase chain reaction (qRT-PCR). QRT-PCR reaction system consisted of $4 \mu \mathrm{l}$ of reverse transcription product, $0.5 \mu \mathrm{l}$ of BlazeTaq ${ }^{\mathrm{TM}} \mathrm{SYBR}^{\circledR}$ Green qPCR Mix 2.0 (GeneCopoeia), $0.5 \mu \mathrm{l}$ each of forward and reverse primers, and $3 \mu \mathrm{l}$ Nuclease-Free Water. PCR was performed in a Mini Amp Thermal Cycller under the following conditions: pre-denaturation at $95^{\circ} \mathrm{C}$ for $30 \mathrm{~s} ; 40$ cycles of denaturation at $95^{\circ} \mathrm{C}$ for $10 \mathrm{~s} ; 40$ cycles of annealing at $60^{\circ} \mathrm{C}$ for $20 \mathrm{~s} ; 40$ cycles of extension for $20 \mathrm{~s}$. The GAPDH protein was served as an internal control. RNA levels were calculated for tumor samples and paired adjacent samples using the $2^{-\Delta \mathrm{Ct}}$ method. Primer sequences used for qRT-PCR are shown in Supplementary Table 2 .

\section{Gene Set Enrichment Analysis}

To better understand the functional pathways of the high- and low-risk groups, we used GSEA to perform Gene Ontology (GO) and the Kyoto Encyclopedia of Genes and Genomes (KEGG) enrichment analyses. GSEA was performed by using clusterProfiler. $P<0.05$ was considered statistically significant.

\section{Mutation Analysis}

The somatic mutation data of the 112 TNBC samples were obtained from the TCGA database. We used the "maftools" tool to comprehensively analyze mutation status in TNBC. The "somaticInteractions" function in the R package "maftools" was used to perform a Fisher test on the mutated genes in order to obtain the interaction relationships between them.

\section{Gene Expression in Pan-Cancer}

The expression of six prognostic genes in Pan-Cancer was analyzed by TIMER $2.0^{4}$, which integrates multiple heterogeneous types of data, including gene symbol, name and location.

${ }^{4} \mathrm{http} / /$ timer.cistrome.org/ 


\section{Immune Analysis}

The enrichment levels of 28 immune signatures in each TNBC sample were quantified by single-sample gene set enrichment analysis (ssGSEA) in the R package "GSVA." Heat maps and violin plots were drawn to observe the difference in the level of various immune cell infiltration between the high- and low-risk groups. Finally, the correlation between 6 genes and 28 immune signatures was calculated by the Spearman method.

\section{Immunohistochemistry and Protein Level Validation}

The Human Protein Atlas (HPA) provides information on the tissue and cell distributions of 26,000 human proteins (Uhlén et al., 2015, Uhlen et al., 2017; Thul et al., 2017). We used the HPA database to detect the protein expression level of six prognostic genes by immunohistochemistry (IHC) staining, and obtained IHC images from the HPA database.

\section{Transcription Factors and ceRNA Network Construction}

The Network analyst database ${ }^{5}$ is an online visual analysis platform for gene expression analysis and meta-analysis. In this study, the Network analyst database was used to search the transcriptional factors (TFs) related to the hub genes, which refers to the genes used to construct the prognostic model.

The mRNA-miRNA and miRNA-lncRNA interactions were predicted by using the miRanda database ${ }^{6}$. To improve the reliability of the competing endogenous RNA (ceRNA) network, we used a binding score $>500$ and minimal folding free energy $(\mathrm{MFE})<-50$ for the predicted mRNA-target miRNA interaction. The screening criteria of miRNA-lncRNA were as follows: binding score $>4,000$ and $\mathrm{MFE}<-400$.

\section{Statistical Analysis}

All analyses were performed using the $\mathrm{R}$ software. Univariate and LASSO Cox regression analyses were used to assess the relationship between the prognostic model and OS. The KaplanMeier (K-M) method was used to assess survival analysis with a log-rank test. The ROC curves were used to detect the sensitivity and specificity of the prognostic model. $P<0.05$ was considered statistically as significant.

\section{RESULTS}

\section{Identification of DE-DDRs}

Following analysis of the TCGA database using limma, a total of 2,178 DEGs were identified in 113 TNBC and 99 normal samples ( $\mid \log _{2}$ (fold change) $\mid>1, P<0.05$ ). Figure 1A illustrates the 946 up-regulated and 1,232 down-regulated genes using a volcano plot. As shown by the Venn diagrams in Figure 1B, we selected 23 significant DE-DDRs (the intersection of 154 DDRs and 2,178 DEGs) for subsequent analysis. GO function annotation of the

${ }^{5}$ http://www.networkanalyst.ca/faces/home.xhtml

${ }^{6} \mathrm{http}: / /$ www.microrna.org/microrna/home.do
DE-DDRs was performed by the $\mathrm{R}$ package. These genes were mainly enriched in DNA replication, DNA recombination, and DNA-dependent DNA replication (Figure 1C).

\section{Construction of Prognostic Model in the TCGA Database}

In order to establish a prognostic model, univariate Cox regression analysis was performed on 23 genes, of which six genes were significantly associated with the OS of TCGA-TNBC (Figure 2A). The regression coefficients of these six genes were calculated via LASSO COX regression analysis (Figure 2B). The prognostic model achieved the best performance when the six genes were used (Figure 2C). The formula of the model was: risk score $=(-0.18330185 \times$ expression level of PARP1 $)+$ $(0.25938239 \times$ expression level of BRIP 1$)+(-0.71002582 \times$ expression level of RMI2) $+(-0.05379813 \times$ expression level of RFC4 $)+(-1.01590214 \times$ expression level of EXO1) + $(1.46313437 \times$ expression level of RAD51). According to the median risk score, 56 of the 112 TNBC samples were classified into the high-risk group $(n=56)$, and the remaining 56 samples were classified into the low-risk group ( $n=56$; Figure 2D). Survival analysis indicated that the OS was lower in the highrisk group than the low-risk group $(P<0.05$; Figure $2 \mathrm{E})$. The time-dependent ROC curves were used to evaluate the prediction effect of the risk score, and the AUC was 0.821 at 3 years and 0.745 at 5 years (Figure 2F). The relationships between risk score and clinicopathological features (age, sex, pathological stage, and TNM stage) are shown in Figure 2G.

\section{Validation of Prognostic Genes Based on Clinical Samples}

Quantitative real-time polymerase chain reaction analysis were performed to assess the expression levels of the six DEGs that constructed our prognostic model. Consistent with the results of the bioinformatics analysis, all DEGs were identified as significantly up-regulated in the tumor samples compared to the normal samples (Figure 3). The results of qRT-PCR analysis were showed in Table 1.

\section{Validation of the Six-Gene Prognostic Model in the GEO Database}

In order to verify the robustness of the prognostic model, we applied the model to the GEO cohort for external validation. Patients in the GSE58812 dataset $(n=107)$ were divided into a high-risk group $(n=53)$ and low-risk group $(n=54)$ using the formula obtained from TCGA-TNBC cohort (Figure 4A). Consistent with the TCGA cohort, the survival probability of high-risk patients was significantly lower than that of low-risk patients (Figure 4B). As shown in Figure 4C, the AUC of the ROCs was 0.574 for 3 years and 0.663 for 5 years. Since there were only three patients with data regarding the 1-year follow-up, we did not plot the ROC curve of the 1-year follow-up for the TCGA and GSE58812 datasets. Collectively, these results indicated that the six-gene prognostic model was robust for survival prediction. 


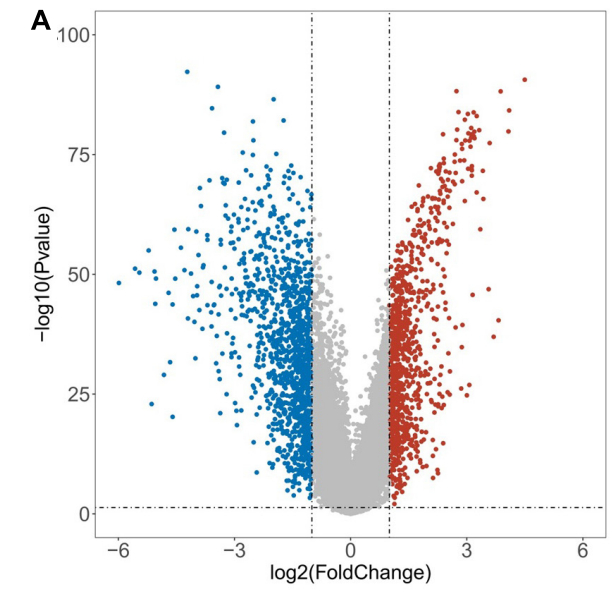

B DEG

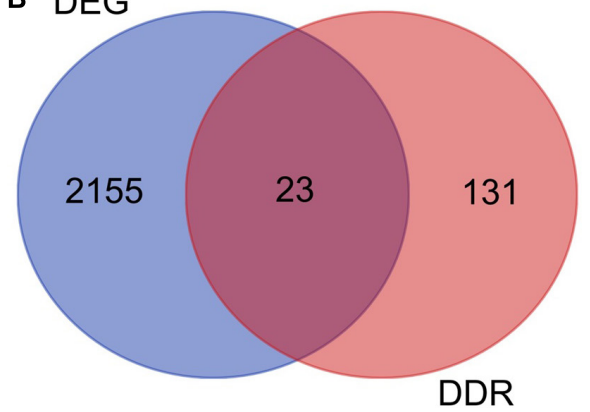

C

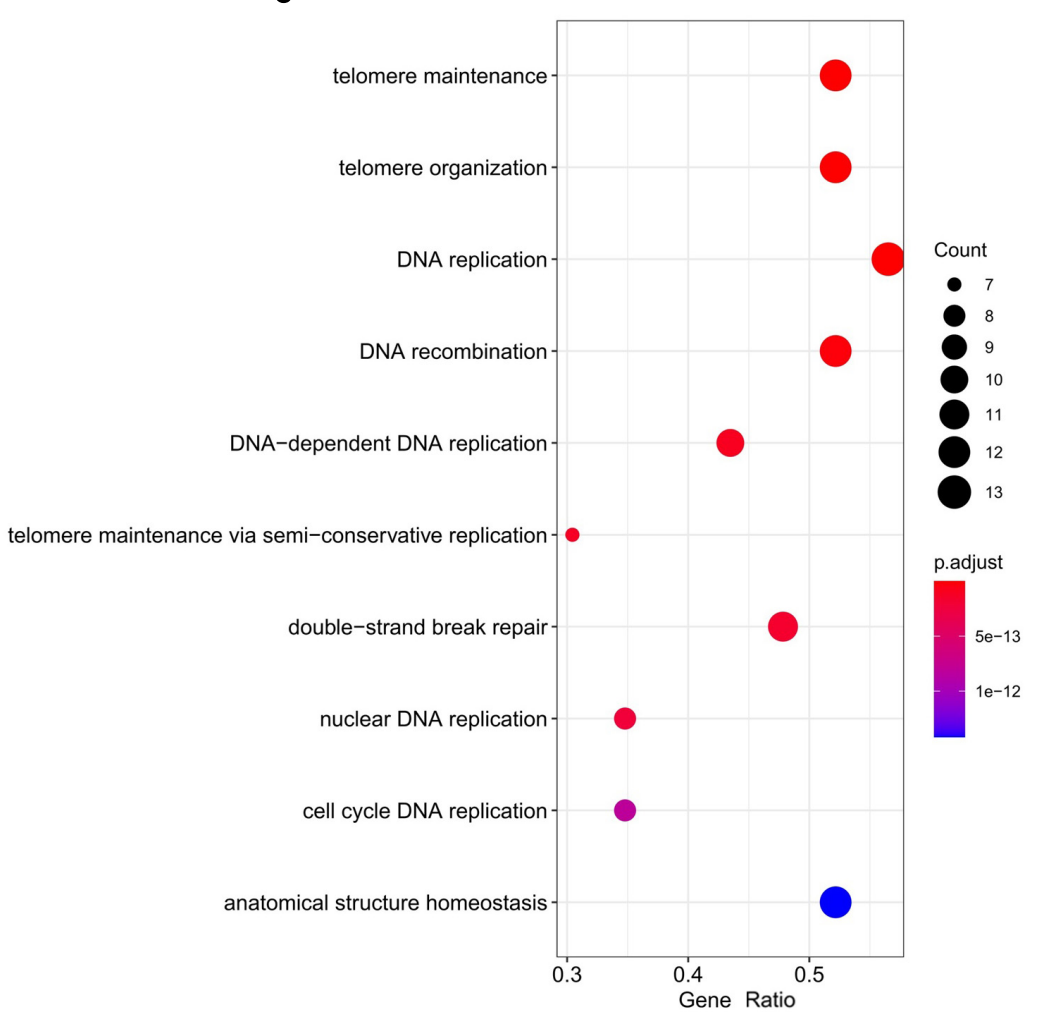

FIGURE 1 | Bioinformatics analysis of the expression of DE-DDRs. (A) A Volcano plot illustrating the comparison of differential gene expression in tumors vs. normal tissue. The red dots indicate that the gene expression level is up-regulated (tumor samples relative to normal samples), the blue dots indicate that the gene expression level is down-regulated (tumor samples relative to normal samples), and the gray dots indicate that there is no significant difference between these genes. (B) A Venn diagram representation showing the intersection of DDRs and DEGs. (C) The results of the GO annotation of DE-DDRs.

\section{Independent Prognostic Value of the Six-Gene Prognostic Model}

Next, we used univariate and multivariate Cox regression analyses to evaluate whether the six-gene prognostic model could serve as an independent predictor for TNBC patients. Univariate Cox regression analysis showed the variables of tumor stage, TNM stage, and risk score were significantly associated with OS $(P<0.05$; Figure 5A). Multivariate Cox regression analysis indicated that $\mathrm{N}$ stage, $\mathrm{T}$ stage, and risk score were independent risk factors correlated with OS $(P<0.05$; Figure 5B). Moreover, a nomogram was constructed to predict the possibility of 3-year and 5-year OS in TNBC patients by integrating the six-gene prognostic model with other clinicopathological characteristics ( $\mathrm{T}$ and $\mathrm{N}$ stage). As shown in Figures $\mathbf{5 C}, \mathbf{D}$, the nomogram and calibration curve demonstrated that the six-gene prognostic model was a valuable indicator for prognostic prediction.

\section{Separate Survival Analysis of the Six DEGs in TNBC}

In TCGA data, we analyzed the effects of the six DEGs on the OS of TNBC patients, but none of them had significant effect on the OS (overall survival) of TNBC patients (Supplementary Figure 1). The results were shown in Supplementary Table 3.

\section{Expression of Six DEGs in 33 Pan-Cancers}

Using the TIMER 2.0 database, we analyzed the expression levels of these six genes at the pan-cancer level, and the results were as follows: BIRP1, PARP1, and RFC4 were significantly overexpressed in BRCA (Breast cancer), BLCA (Bladder Urothelial Carcinoma) and LIHC (Liver hepatocellular carcinoma) while they were significantly lower expressed in $\mathrm{KICH}$ (Kidney Chromophobe). Compared with normal samples, EXO1, RMI2, and RAD51 were significantly overexpressed in most cancer types including BRCA, while RMI2 was significantly lower expressed in PARD (Prostate adenocarcinoma; Supplementary Figure 2).

\section{Gene Set Enrichment Analyses}

We performed GSEA to identify 672 GO terms and 30 KEGG pathways associated with the high- and low-risk groups in the TCGA cohort $(P<0.05)$. As shown in Figures $\mathbf{6 A}$, B and Supplementary Table 4, the genes in the high-risk group were mainly enriched in leukocyte migration, cytokine interaction with cytokine receptors, oxidative phosphorylation, autoimmune diseases, and coagulation cascade. The genes in the low-risk group were mainly enriched in ATPase activity, 


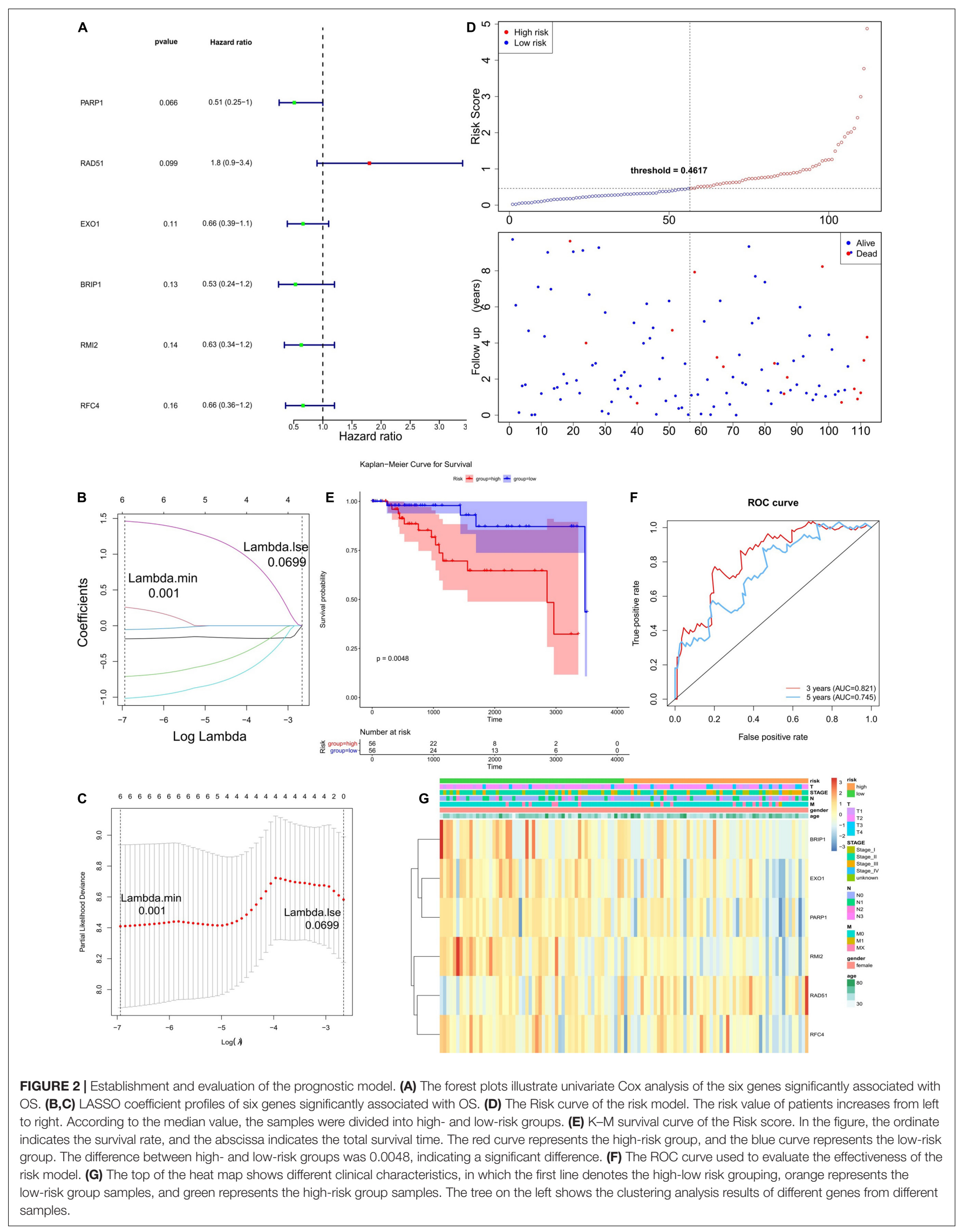



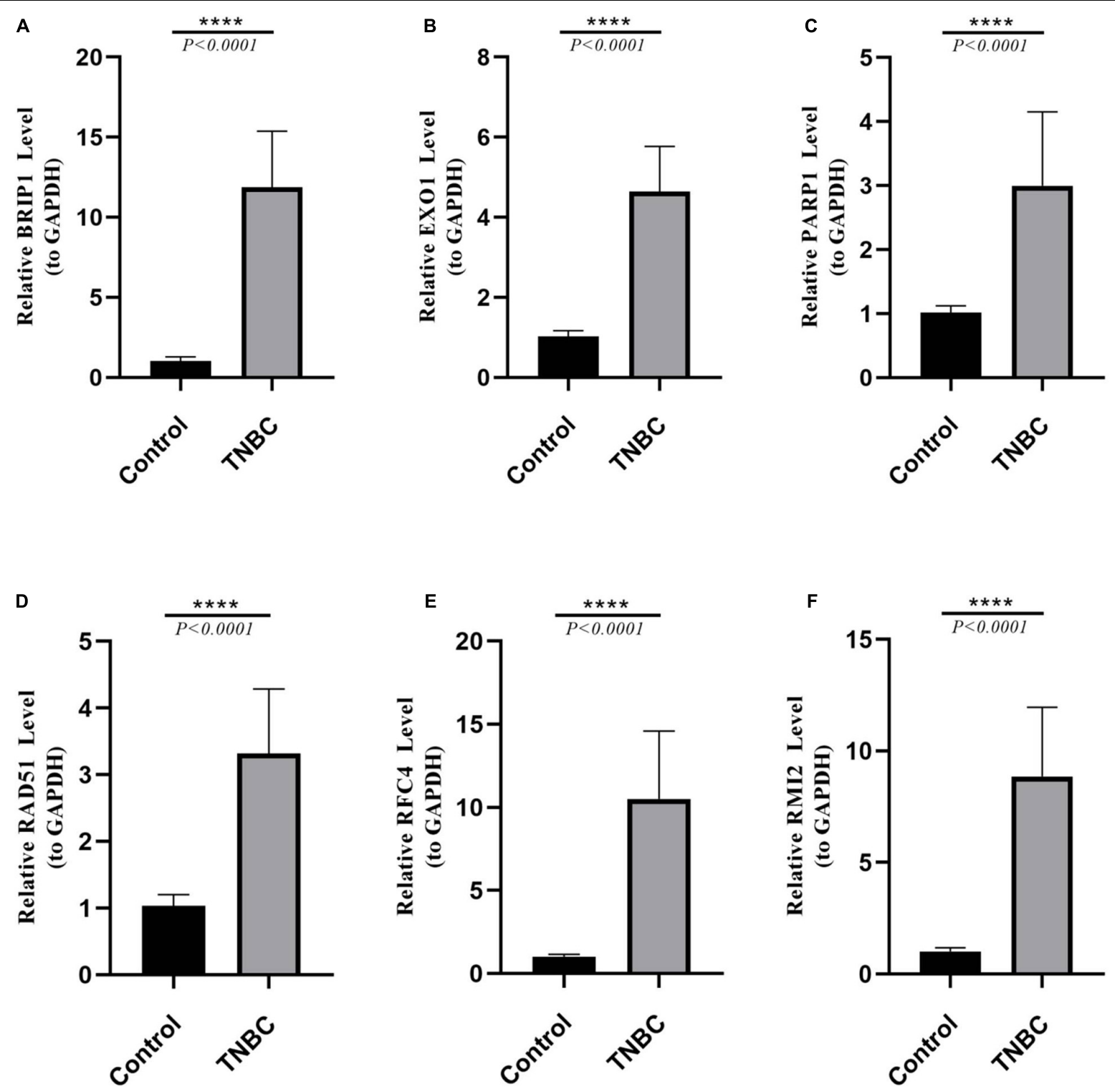

FIGURE 3 | Results of quantitative real-time PCR (qRT-PCR) validation of six differentially expressed genes (DEGs) in TNBC tumor tissues (TNBC) and adjacent normal tissues. The expression of each of these six DEGs was significantly increased in tumor tissues $(P<0.0001)$. Expression levels measured by qRT-PCR analysis of (A) PARP1. (B) RAD51. (C) EXO1. (D) BRIP1. (E) RMI2. (F) RFC4.

chromatin remodeling, DNA replication, methylation, and cell cycle (Figures 6C,D and Supplementary Table 5). In summary, the enrichment analysis revealed potential pathways that could serve as targets in TNBC treatment.

\section{Clinical Validation of Six Genes in Terms of Protein Expression}

The HPA version $9.0^{7}$ is a public database with millions of immunohistochemical images and was used to compare protein expressions between normal and tumor tissues. Since BRCA data were not classified according to molecular subtypes

${ }^{7} \mathrm{http}: / /$ www.proteinatlas.org in HPA, we analyzed the IHC staining of these six DEGs in BRCA to verify the expression level of them. We only found 5 DEGs (BIRP1, PARP1, RFC4, RMI2, and RAD51) had protein expression data in HPA and the results showed that the expression levels of BIRP1, PARP1, RFC4, RMI2, and RAD51 in BRCA tumor tissues were higher than in normal tissues (Figure 7).

\section{Landscape of Mutation Profiles in Low-and High-Risk Groups}

Since the DDR is closely related to somatic mutations (Tian et al., 2020), we further explored the mutation status of the high- and low-risk groups. After analyzing the mutation data, 
TABLE 1 | Results of quantitative real-time PCR (qRT-PCR).

\begin{tabular}{|c|c|c|c|c|}
\hline & Control & TNBC & $t$ & $P$ \\
\hline RAD51 & $1.032 \pm 0.162$ & $3.320 \pm 0.914$ & $t=7.394 \mathrm{df}=18$ & $<0.0002$ \\
\hline BRIP1 & $1.046 \pm 0.235$ & $11.875 \pm 3.326$ & $t=9.742 \mathrm{df}=18$ & $<0.0004$ \\
\hline RMI2 & $1.024 \pm 0.153$ & $8.855 \pm 2.942$ & $t=7.974 \mathrm{df}=18$ & $<0.0005$ \\
\hline RFC4 & $1.020 \pm 0.126$ & $10.503 \pm 3.882$ & $t=7.324 \mathrm{df}=18$ & $<0.0006$ \\
\hline
\end{tabular}

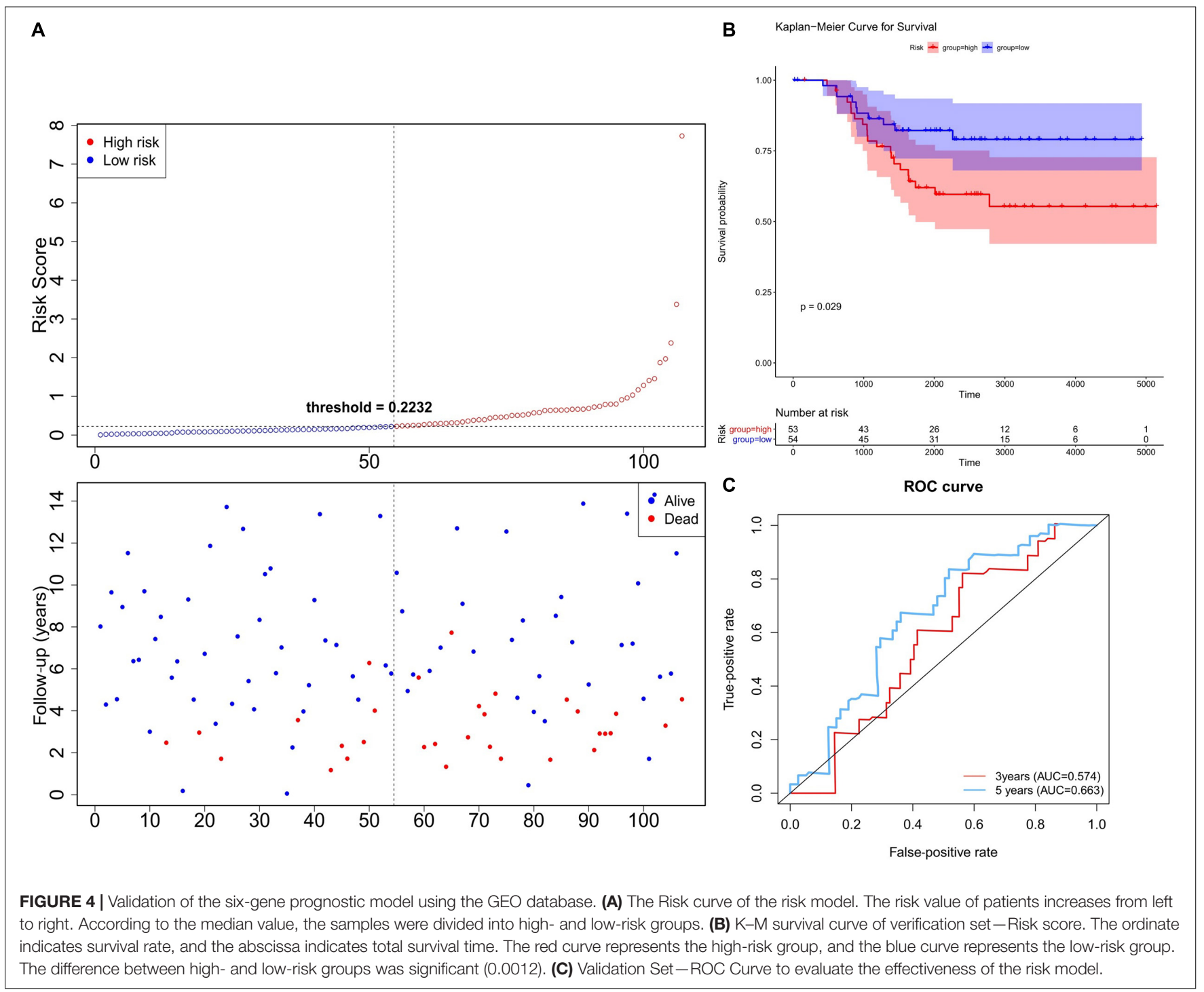

we found missense mutations accounted for the most mutations in the high- and low-risk groups. The main variant type was single-nucleotide polymorphism, with the most common single nucleotide variants being $\mathrm{C}>\mathrm{T}$ (Figures 8A,B). Figures 8C,D show the top 20 most frequently mutated genes in the high- and low-risk groups with ranked percentages. The mutation frequency of the two groups was the same $(94 \%$ vs. 93.75\%), while the mutated genes were different. Additionally, the associations across the top 20 mutated genes are shown in Supplementary Figures 3A,B, where green represents coexpression and red represents mutually exclusive relationships.

\section{Immune Status Between Low- and High-Risk Groups}

To further explore the relationship between the six-gene prognostic model and the immune system, ssGSEA was used to evaluate the expression profiles of 28 immune signature genomes 


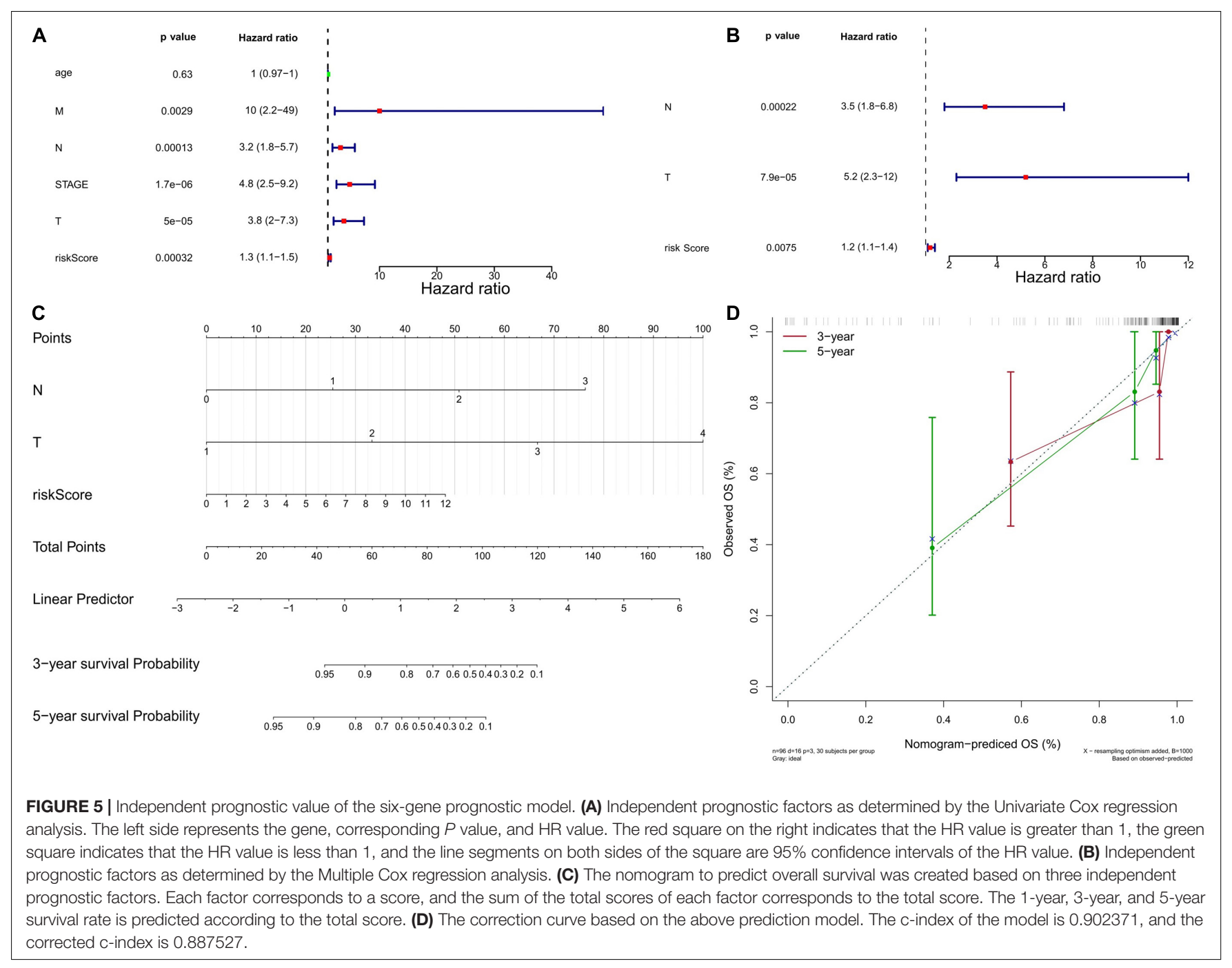

in the high- and low-risk groups. The heat map in Figure 9A shows that in the TCGA database, 28 types of immune cells in the low- and high-risk groups showed a certain degree of heterogeneity. The violin plot of the 28 immune cells showed that, as compared with the low-risk group, the content of memory $\mathrm{B}$ cells and $\mathrm{T}$ follicular helper cells increased, while the content of myeloid-derived suppressor cells (MDSCs) decreased (Figure 9B). We also showed the correlation analysis of six genes and 28 immune cells (Supplementary Figures 4A,B).

\section{Construction of TF and ceRNA Regulatory Network}

A gene-TF regulatory network was constructed using the Network Analyst database for the six-gene prognostic model we developed in this study. As shown in Figure 10A, we were unable to search for TF related to BRIP1 and PARP1. The constructed transcriptional regulatory network included 105 interaction pairs among 4 genes and $87 \mathrm{TFs}$, of which RFC4 regulated most of the TF and occupied the dominant position. Finally, we predicted the target miRNAs of the six-gene prognostic model and lncRNAs that may be related to miRNAs via the miRanda database. A total of 271 lncRNA-miRNA-mRNA pairs were obtained, including 94 lncRNAs, 25 miRNAs, and 3 mRNAs (Figure 10B). It is worth noting that three mRNAs in this ceRNA network including BRIP1 and PARP1, were not found in the Network Analyst database.

\section{DISCUSSION}

DNA is the source of genetic information, and maintaining its integrity is vital to sustaining life. Therefore, cells have evolved specialized DDR mechanisms to maintain the integrity of the genome (Malaquin et al., 2015; Wengner et al., 2020). DDR plays an important role in maintaining homeostasis within the cell (Goldstein and Kastan, 2015). Cancer cells are characterized by genomic instability, which is conducive to the accumulation of driver mutations and the expansion of tumor heterogeneity (Lin et al., 2019; Hu and Guo, 2020). DDR mechanisms can repair mutated genes during the early stage of cancer and hinder the development of tumors (Ali et al., 2017). However, with the development of cancer, DDRs may cause tumor cells to develop 


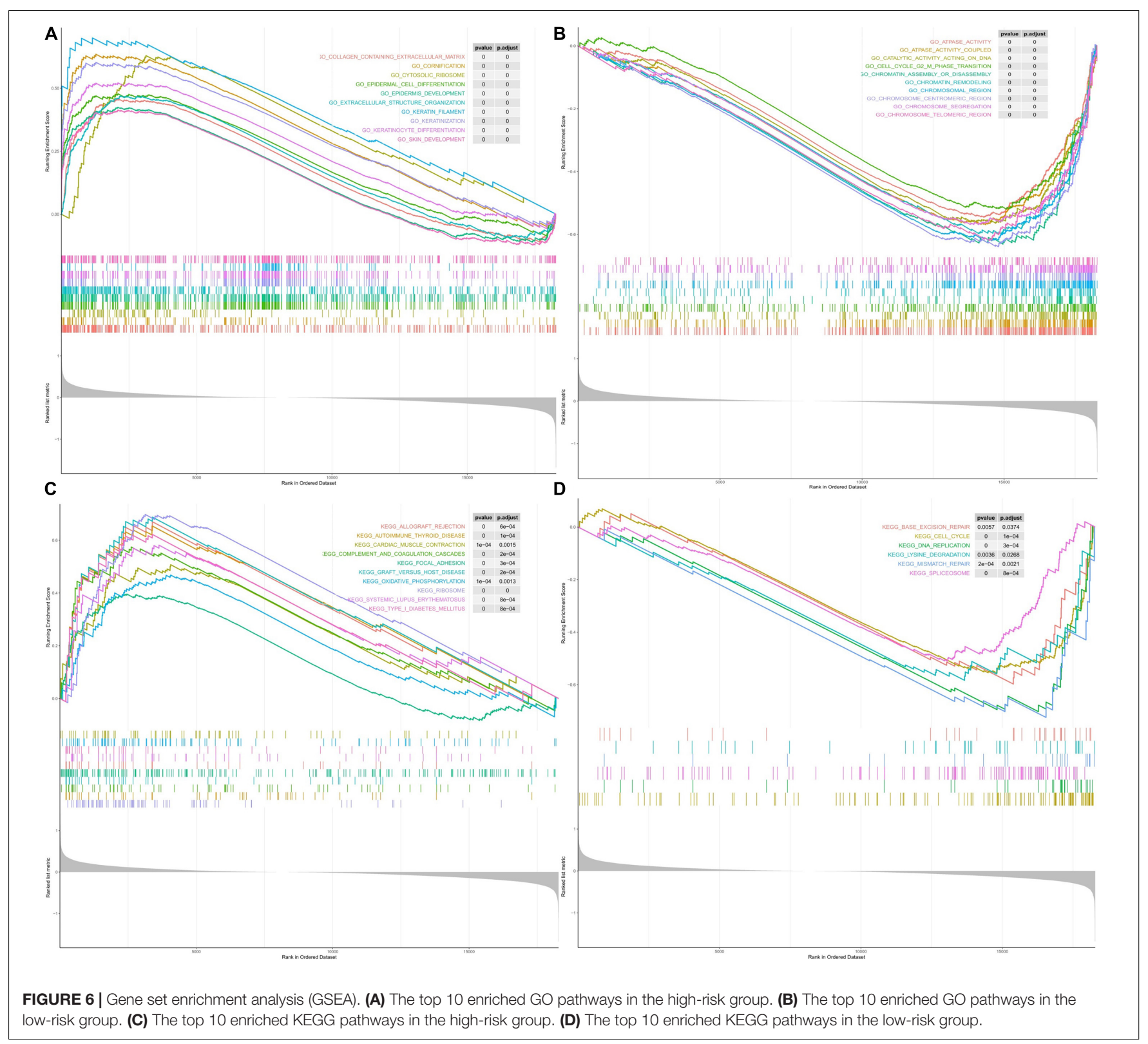

resistance to cytotoxic drugs (Brandsma et al., 2017; Carusillo and Mussolino, 2020). The occurrence and development of cancer are often accompanied by the inactivation of one or more DDR pathways; however, cancer cells are therefore more dependent on the remaining DDR pathways than normal cells (Carusillo and Mussolino, 2020). This phenomenon suggests that there are potential weaknesses in tumors that can be targeted by innovative therapies that follow the concept of synthetic lethality. In the concept of synthetic lethality, two pathway defects (independently non-toxic) become fatal when combined (Blackford and Jackson, 2017; Nickoloff et al., 2017; Pilié et al., 2019; Yap et al., 2019). In this study, we screened out six prognostic genes from DDR genes, constructed a risk model based on the bio-analysis of DDR genes, and conducted immune-related analysis based on the model. Our research provides a theoretical basis and reference for the diagnosis and treatment of TNBC.

From the TNBC patient data in the TCGA database, we obtained 2,178 differential genes and intersected them with DDRs genes to obtain 23 DE-DDRs. We then constructed a risk model based on six prognostic genes (PARP1, RAD51, EXO1, BRIP1, $R M I 2$, and RFC4) using univariate analysis and Lasso analysis and determined the effectiveness of the risk model by drawing an ROC curve and a $\mathrm{K}-\mathrm{M}$ curve. In addition, independent prognostic analysis of the risk model and verification of the model based on the GEO dataset confirmed that the risk model constructed in this study can effectively predict the prognosis of TNBC. In order to investigate the reasons why the model could effectively predict the prognosis of TNBC patients, we conducted enrichment analysis, mutation analysis, and immunocorrelation 


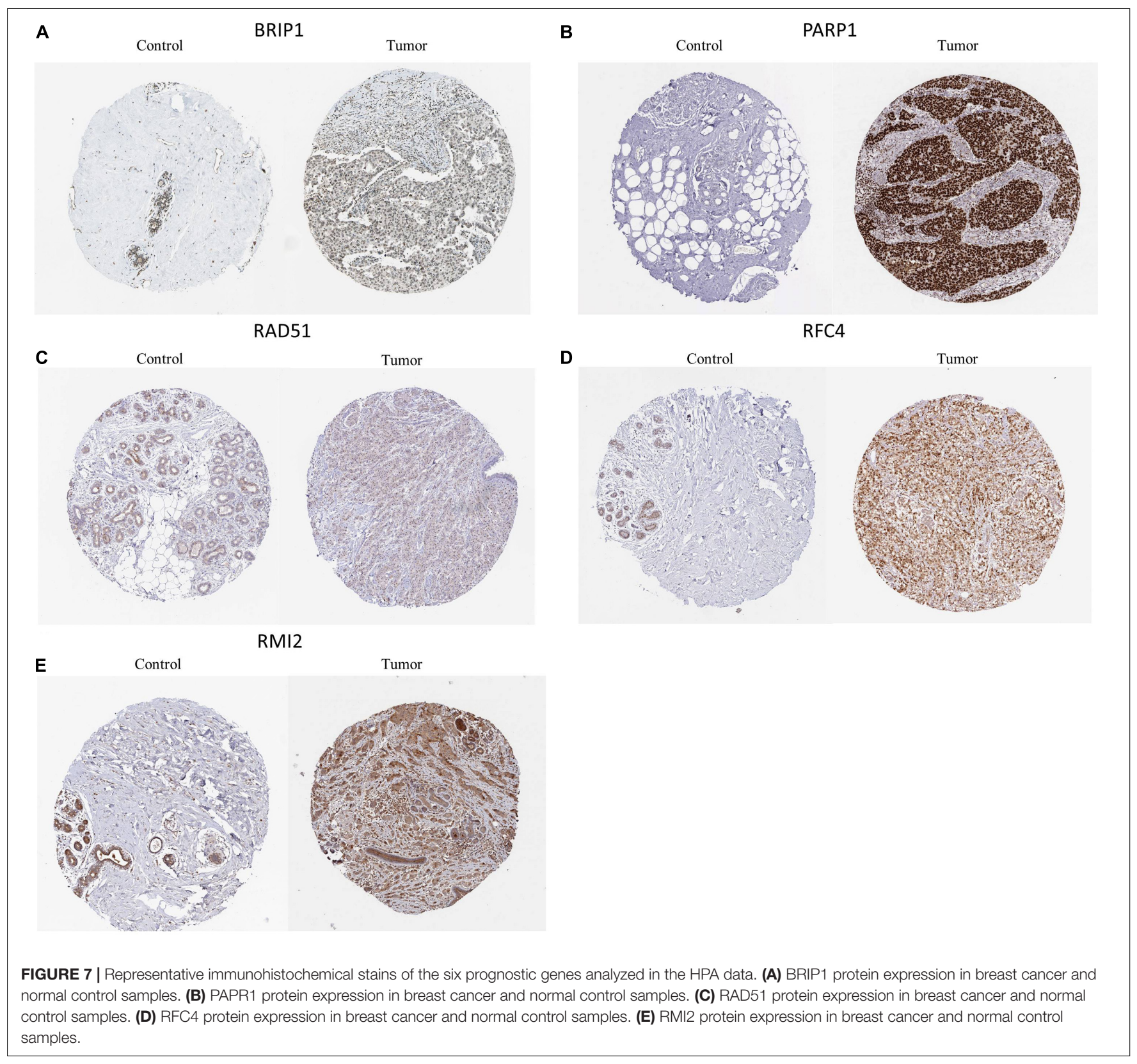

analysis (immunoinfiltration and immunocorrelation score) on the high- and low-risk groups defined by the model. We found that there were certain differences in immunity between the highand low-risk groups. Finally, we constructed a TF regulatory network and ceRNA network based on model gene prediction and demonstrated the regulatory function of these key genes.

Poly(ADP-ribose) Polymerase-1 (PARP1) is a member of the PARP family, which has 17 members total and plays a role in various biological functions, including synthetic lethality, DNA repair, apoptosis, necrosis, and histone binding. PARP1, a chromatin-bound nuclear enzyme that is activated by DNA damage, is a validated therapeutic target for cancers and other human diseases (Jain and Patel, 2019; Cao et al., 2020). PARP1 can inhibit the expression of PD-L1 on the surface of TNBC cells by interacting with the nucleic acid-binding domain of nucleophosmin, thus playing a key role in the tumor-related immune escape of TNBC (Qin et al., 2020). In our study, we found that the PARP1 gene correlated positively with eosinophils.

RAD51 is a strand transferase that aggregates into nucleoprotein filaments on single strands of DNA and promotes the exchange of DNA strands with undamaged homologous chromatin (San Filippo et al., 2008). RAD51 is a component of the cellular DDR, and as such, inhibition of RAD51 sensitizes cancer cells to DNA-damaging drugs (Tsai et al., 2010; Quiros et al., 2011). Studies have found that RAD51 can mediate breast cancer stem cells to develop resistance to PARP inhibitors in TNBC (Liu et al., 2017). In our study, we found a negative correlation between the RAD51 gene and immune dense cells. 


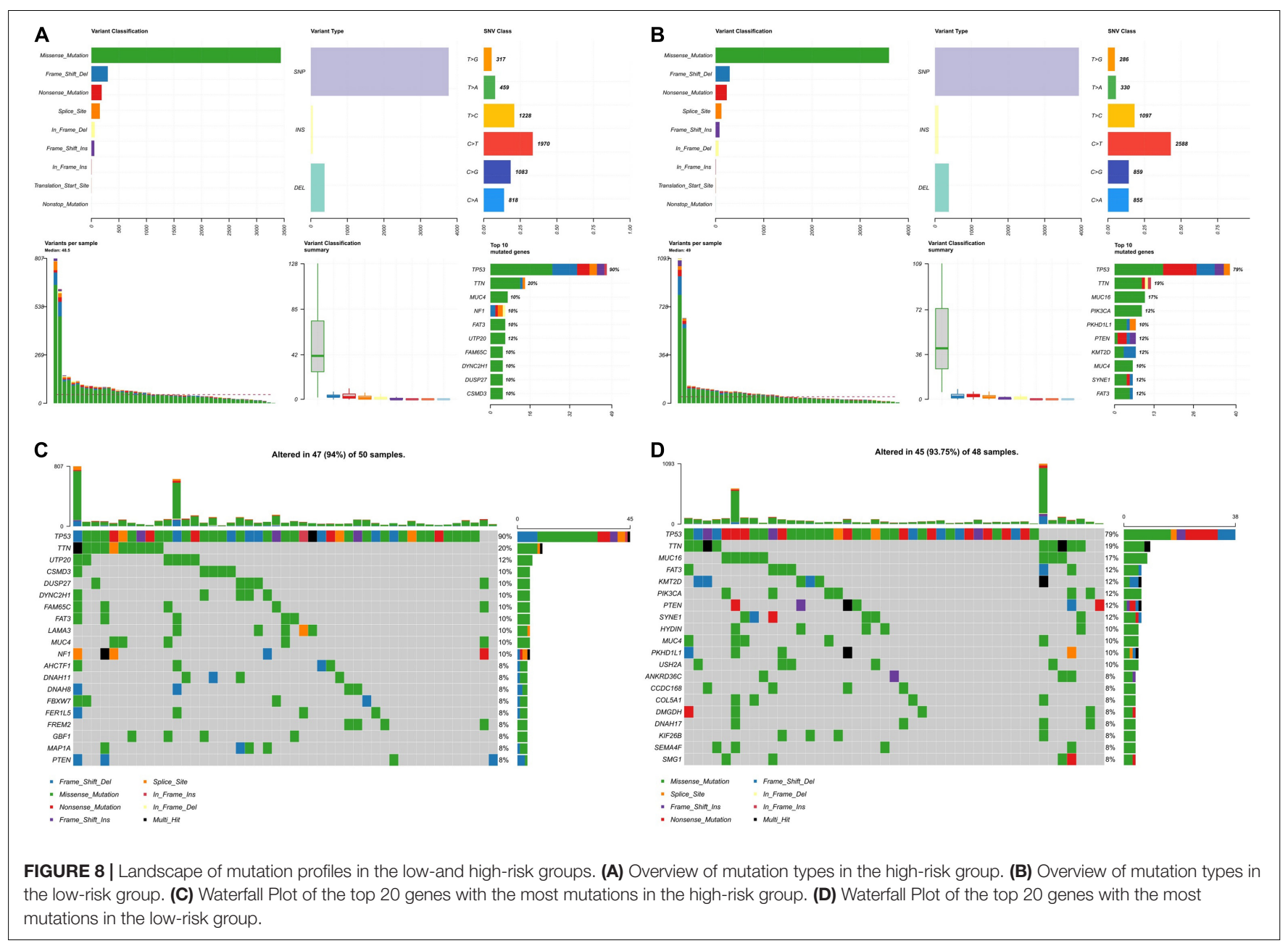

BRIP1, which belongs to the Fanconi anemia (FA) gene family, was first identified via tandem mass spectrometry through its physical interaction with BRCA1 (Rutter et al., 2003). BRIP1 is essential to the stability of the genome, and its normal active expression is necessary for the repair of DNA interstrand crosslinks (Moyer et al., 2020). Although pathogenic mutations and a large number of missense mutations in BRIP1 have been discovered through genetic testing, the impact of these mutations on the molecular function and subsequent role of BRIP1 in cancer risk is uncertain (Lu et al., 2015; Easton et al., 2016; Weber-Lassalle et al., 2018). Studies have found that BRIP1 can promote the invasion of breast cancer (BC) cells by regulating the expression of multiple downstream target genes, such as MGAT5, EPCAM, and CXCL12, especially in the triple-negative phenotype MDA-MB-231 cell line (Rizeq et al., 2020). In our study, we found a positive correlation between the BRIP1 gene and monocytes.

Exonuclease 1 (EXO1) is associated with increased levels of genomic instability in the telomere region, and this widespread genomic instability can promote cancer progression (Maciejowski and de Lange, 2017). EXO1 is a therapeutic target of TNBC that serves an important role in the DDR by inhibiting the activity of PARP (Quist et al., 2019; Li et al., 2021). In our study, we found that the EXO1 gene has a positive correlation with eosinophils.

RecQ-mediated genome instability protein 2 (RMI2) plays a vital role when the spindle assembly point (Battaglia et al., 2020) is activated during mitosis (Pradhan et al., 2013). RMI2 is widely considered to play a crucial role in DNA damage repair. High expression of RMI2 was confirmed to be associated with the worse prognosis in pancreatic cancer (Xu et al., 2018) and lung cancer (Zhan et al., 2020). RMI2 was also reported to act as a tumor promoter by mediating MYCN/PARP DDR signaling pathway in neuroendocrine prostate cancer (Zhang et al., 2018).

Human Replication Factor C (RFC) is a polyprotein composed of five distinct subunits that are highly conserved through evolution and plays an important role in DNA repair after DNA damage (Kim and Brill, 2001; Krause et al., 2001). Human replication factor $\mathrm{C}$ subunit 4 (RFC4) is a member of the RFC family that is often overexpressed in cancer, promoting tumor progression and resulting in worse survival outcomes by regulating tumor cell proliferation and cell cycle. RFC4 has been reported to be overexpressed in a variety of malignancies, including prostate cancer, cervical cancer, colorectal cancer, and head and neck squamous cell carcinoma (Slebos et al., 2006; Narayan et al., 2007; Erdogan et al., 2009; Kang et al., 2009). It can 

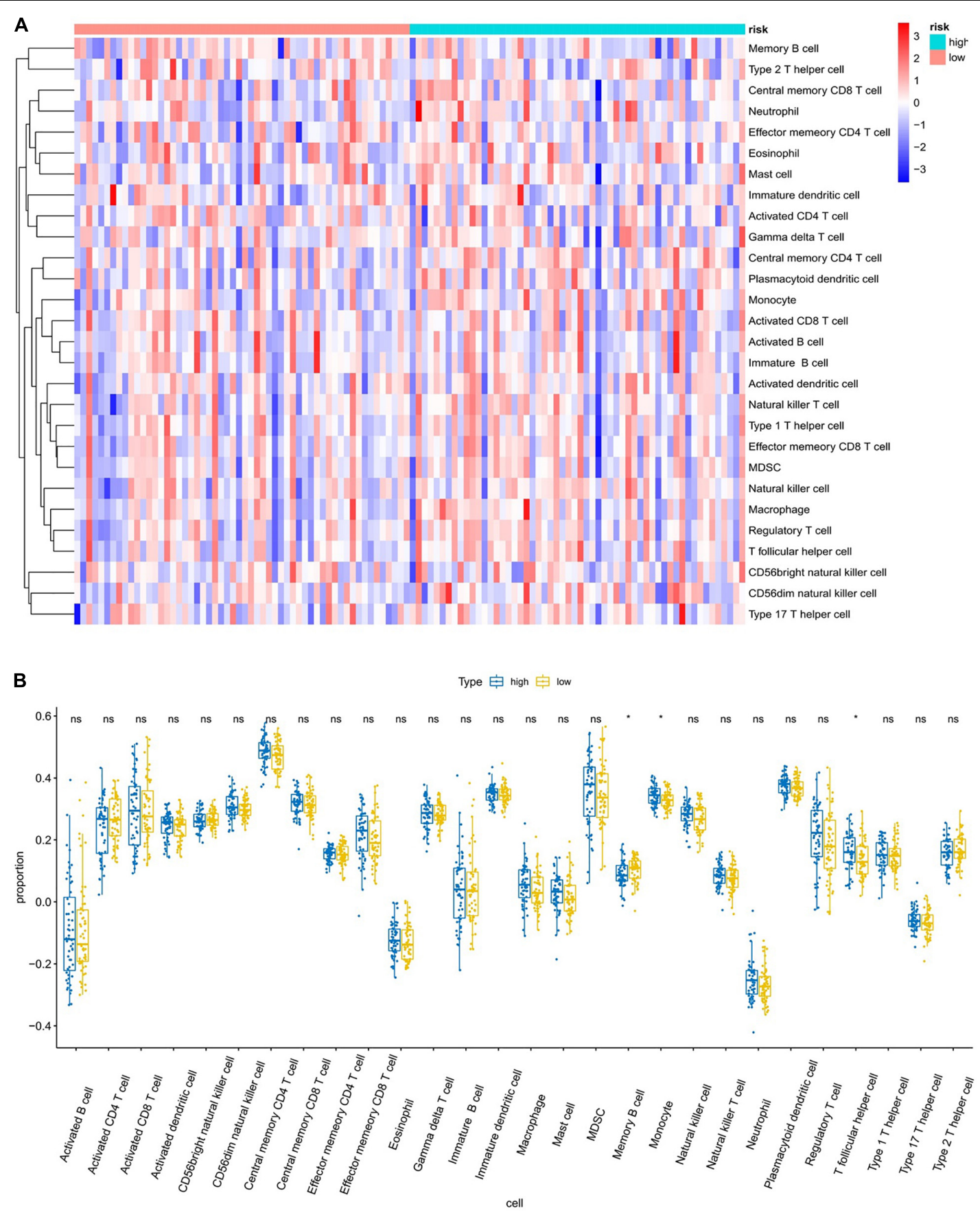

FIGURE 9 | Analysis of immune infiltration patterns in breast cancer samples from TCGA dataset via an ssGSEA score-based method. "ns" represents that there is no significant difference in the infiltration of immune cells between the two samples; "*” represents $P<0.05$. (A) Heatmap of ssGSEA scores (red = positive, blue $=$ negative). (B) Boxplot of ssGSEA scores of the 28 representative gene sets.

promote tumor progression and lead to worse survival outcomes by regulating cell proliferation and cell cycle arrest in tumors (Yao and O'Donnell, 2012). In our study, we found that the RFC4 gene had a positive correlation with type $2 \mathrm{~T}$ helper cells and a negative correlation with mast cells.

Our results demonstrate that further elucidating the functions of these six DDR-related genes in TNBC may improve our understanding of the biological basis of breast cancer and provide new therapeutic targets. The poor prognosis of TNBC seems to depend on the multi-layered interaction between DNA repair gene mutations, cell proliferation, and the immune response. By including prediction-related biological features, such as immune cells, our six-gene model displayed better predictive value than previously published immune features. 

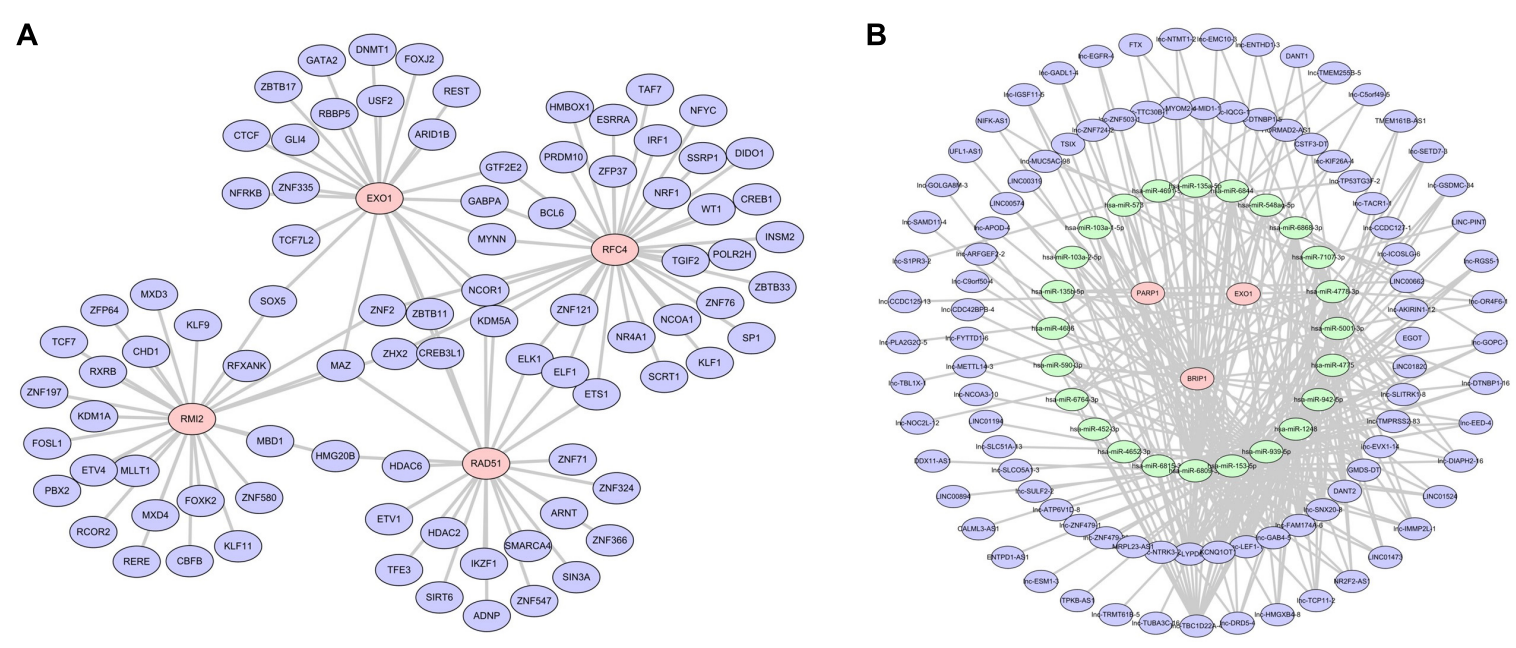

FIGURE 10 | Regulatory network of the risk model genes. (A) Transcription factor regulation network diagram. Only four of the six model genes (RAD51, EXO1, $R M I 2$, and RFC4) predicted the corresponding transcription factors. In the picture, red is the model gene and blue is TF. (B) Transcription factor regulation network diagram. In the picture, red is the model gene, green is miRNA, and blue is IncRNA. Three model genes, 25 miRNAs, and 94 IncRNAs were screened out.

In this study, the correlation between our six gene markers related to the DDR and immune characteristics has been characterized to a certain extent. This model outperforms the prognostic performance of individual clinicopathological prognostic factors and published markers of disease-free survival gene expression, further reinforcing the fact that the immune response is an important component of TNBC. Analyzing the function of the six genetic signatures not only helped us to understand the biological basis of the risk association, but also aided us in making treatment decisions. The main limitations of this study are the retrospective nature of the study and the genes included in the signature were only initially verified by qRT-PCR. More functional validation will be further verified in future experiments and prospective studies.

\section{CONCLUSION}

We screened and identified six DE-DDRs (PARP1, RAD51, EXO1, BRIP1, RMI2, and RFC4) as prognostic genes through comprehensive bioinformatics analysis and constructed a risk model that can effectively predict the prognosis of TNBC. In addition, we found that the high- and low risk TNBC groups, as defined by the model, exhibited differences in immunerelated analysis (immune infiltration, immune-related scores). The above analysis provides a theoretical basis and reference for the research and treatment of TNBC.

\section{DATA AVAILABILITY STATEMENT}

The datasets presented in this study can be found in online repositories. The names of the repository/repositories and accession number(s) can be found in the article/Supplementary Material.

\section{ETHICS STATEMENT}

The studies involving human participants were reviewed and approved by Ethics Committee of the First Affiliated Hospital of Zhengzhou University. Written informed consent for participation was not required for this study in accordance with the national legislation and the institutional requirements.

\section{AUTHOR CONTRIBUTIONS}

NW and YX performed the data analysis and wrote the manuscript. JC, XL, and CZ contributed to the data analysis and manuscript revision. FW and XW contributed to literature search and data extraction. LL and YG proofread the manuscript. NW and LL conceived and designed the study. All authors contributed to the article and approved the submitted version.

\section{FUNDING}

Science and Technology Department of Henan Province; Henan Medical Science and Technology Joint Building Program (Award Number: LHGJ20200356); Recipient: NW.

\section{ACKNOWLEDGMENTS}

We thank LetPub (www.letpub.com) for its linguistic assistance during the preparation of this manuscript.

\section{SUPPLEMENTARY MATERIAL}

The Supplementary Material for this article can be found online at: https://www.frontiersin.org/articles/10.3389/fgene.2021. 721873/full\#supplementary-material 


\section{REFERENCES}

Ali, R., Rakha, E. A., Madhusudan, S., and Bryant, H. E. (2017). DNA damage repair in breast cancer and its therapeutic implications. Pathology 49, 156-165. doi: $10.1016 /$ j.pathol.2016.11.002

Allemani, C., Matsuda, T., Di Carlo, V., Harewood, R., Matz, M., Nikšić, M., et al. (2018). Global surveillance of trends in cancer survival 2000-14 (CONCORD3): analysis of individual records for 37513025 patients diagnosed with one of 18 cancers from 322 population-based registries in 71 countries. Lancet 391, 1023-1075. doi: 10.1016/s0140-6736(17)33326-3

André, F., and Zielinski, C. C. (2012). Optimal strategies for the treatment of metastatic triple-negative breast cancer with currently approved agents. Ann. Oncol. 23(Suppl. 6), vi46-vi51. doi: 10.1093/annonc/mds195

Battaglia, A. M., Chirillo, R., Aversa, I., Sacco, A., Costanzo, F., and Biamonte, F. (2020). Ferroptosis and cancer: mitochondria meet the "Iron Maiden" cell death. Cells 9:1505. doi: 10.3390/cells9061505

Bianchini, G., Balko, J. M., Mayer, I. A., Sanders, M. E., and Gianni, L. (2016). Triple-negative breast cancer: challenges and opportunities of a heterogeneous disease. Nat. Rev. Clin. Oncol. 13, 674-690. doi: 10.1038/nrclinonc. 2016.66

Blackford, A. N., and Jackson, S. P. (2017). ATM, ATR, and DNA-PK: the trinity at the heart of the DNA damage response. Mol. Cell. 66, 801-817. doi: 10.1016/j. molcel.2017.05.015

Brandsma, I., Fleuren, E. D. G., Williamson, C. T., and Lord, C. J. (2017). Directing the use of DDR kinase inhibitors in cancer treatment. Expert Opin. Investig. Drugs 26, 1341-1355. doi: 10.1080/13543784.2017.1389895

Cancer Genome Atlas Research Network (2017). Integrated genomic characterization of pancreatic ductal adenocarcinoma. Cancer Cell 32, 185.e13-203.e13. doi: 10.1016/j.ccell.2017.07.007

Cao, C., Yang, J., Chen, Y., Zhou, P., Wang, Y., Du, W., et al. (2020). Discovery of SK-575 as a highly potent and efficacious proteolysis-targeting chimera degrader of PARP1 for treating cancers. J. Med. Chem. 63, 11012-11033. doi: 10.1021/acs.jmedchem.0c00821

Carusillo, A., and Mussolino, C. (2020). DNA damage: from threat to treatment. Cells 9:1665. doi: 10.3390/cells9071665

Cerrato, A., Morra, F., and Celetti, A. (2016). Use of poly ADP-ribose polymerase [PARP] inhibitors in cancer cells bearing DDR defects: the rationale for their inclusion in the clinic. J. Exp. Clin. Cancer Res. 35:179. doi: 10.1186/s13046016-0456-2

Easton, D. F., Lesueur, F., Decker, B., Michailidou, K., Li, J., Allen, J., et al. (2016). No evidence that protein truncating variants in BRIP1 are associated with breast cancer risk: implications for gene panel testing. J. Med. Genet. 53, 298-309. doi: 10.1136/jmedgenet-2015-103529

Emens, L. A. (2018). Breast cancer immunotherapy: facts and hopes. Clin. Cancer Res. 24, 511-520. doi: 10.1158/1078-0432.Ccr-16-3001

Erdogan, E., Klee, E. W., Thompson, E. A., and Fields, A. P. (2009). Meta-analysis of oncogenic protein kinase Ciota signaling in lung adenocarcinoma. Clin. Cancer Res. 15, 1527-1533. doi: 10.1158/1078-0432.Ccr-08-2459

Faraoni, I., and Graziani, G. (2018). Role of BRCA mutations in cancer treatment with Poly(ADP-ribose) polymerase (PARP) inhibitors. Cancers 10:487. doi: 10. 3390/cancers 10120487

Goldstein, M., and Kastan, M. B. (2015). The DNA damage response: implications for tumor responses to radiation and chemotherapy. Annu. Rev. Med. 66, 129-143. doi: 10.1146/annurev-med-081313-121208

Hu, Y., and Guo, M. (2020). Synthetic lethality strategies: beyond BRCA1/2 mutations in pancreatic cancer. Cancer Sci. 111, 3111-3121. doi: 10.1111/cas. 14565

Jain, P. G., and Patel, B. D. (2019). Medicinal chemistry approaches of poly ADPRibose polymerase 1 (PARP1) inhibitors as anticancer agents-A recent update. Eur. J. Med. Chem. 165, 198-215. doi: 10.1016/j.ejmech.2019.01.024

Kang, B. Y., You, H., Bandyopadhyay, S., Agrawal, N., Melchert, R. B., Basnakian, A. G., et al. (2009). Cervical cancer isolate PT3, super-permissive for adeno-associated virus replication, over-expresses DNA polymerase delta, PCNA, RFC and RPA. BMC Microbiol. 9:79. doi: 10.1186/1471-21 80-9-79

Kang, S. J., Cho, Y. R., Park, G. M., Ahn, J. M., Han, S. B., Lee, J. Y., et al. (2013). Predictors for functionally significant in-stent restenosis: an integrated analysis using coronary angiography, IVUS, and myocardial perfusion imaging. JACC Cardiovasc. Imaging 6, 1183-1190. doi: 10.1016/j.jcmg.2013.09.006

Kim, H. S., and Brill, S. J. (2001). Rfc4 interacts with Rpal and is required for both DNA replication and DNA damage checkpoints in Saccharomyces cerevisiae. Mol. Cell. Biol. 21, 3725-3737. doi: 10.1128/mcb.21.11.3725-3737.2001

Krause, S. A., Loupart, M. L., Vass, S., Schoenfelder, S., Harrison, S., and Heck, M. M. (2001). Loss of cell cycle checkpoint control in Drosophila Rfc4 mutants. Mol. Cell. Biol. 21, 5156-5168. doi: 10.1128/mcb.21.15.5156-5168.2001

Lawrence, M. S., Stojanov, P., Mermel, C. H., Robinson, J. T., Garraway, L. A., Golub, T. R., et al. (2014). Discovery and saturation analysis of cancer genes across 21 tumour types. Nature 505, 495-501. doi: 10.1038/nature12912

Lee, J. S., Yost, S. E., and Yuan, Y. (2020). Neoadjuvant treatment for triple negative breast cancer: recent progresses and challenges. Cancers 12:1404. doi: 10.3390/ cancers 12061404

Li, Y., Wang, Y., Zhang, W., Wang, X., Chen, L., and Wang, S. (2021). BKM120 sensitizes BRCA-proficient triple negative breast cancer cells to olaparib through regulating FOXM1 and Exol expression. Sci. Rep. 11:4774. doi: 10. 1038/s41598-021-82990-y

Lin, J., Shi, J., Guo, H., Yang, X., Jiang, Y., Long, J., et al. (2019). Alterations in DNA damage repair genes in primary liver cancer. Clin. Cancer Res. 25, 4701-4711. doi: 10.1158/1078-0432.Ccr-19-0127

Liu, Y., Burness, M. L., Martin-Trevino, R., Guy, J., Bai, S., Harouaka, R., et al. (2017). RAD51 mediates resistance of cancer stem cells to PARP inhibition in triple-negative breast cancer. Clin. Cancer Res. 23, 514-522. doi: 10.1158/10780432.Ccr-15-1348

Lu, C., Xie, M., Wendl, M. C., Wang, J., McLellan, M. D., Leiserson, M. D., et al. (2015). Patterns and functional implications of rare germline variants across 12 cancer types. Nat. Commun. 6:10086. doi: 10.1038/ncomms 10086

Maciejowski, J., and de Lange, T. (2017). Telomeres in cancer: tumour suppression and genome instability. Nat. Rev. Mol. Cell Biol. 18, 175-186. doi: 10.1038/nrm. 2016.171

Malaquin, N., Carrier-Leclerc, A., Dessureault, M., and Rodier, F. (2015). DDRmediated crosstalk between DNA-damaged cells and their microenvironment. Front. Genet. 6:94. doi: 10.3389/fgene.2015.00094

Mavaddat, N., Michailidou, K., Dennis, J., Lush, M., Fachal, L., Lee, A., et al. (2019). Polygenic risk scores for prediction of breast cancer and breast cancer subtypes. Am. J. Hum. Genet. 104, 21-34. doi: 10.1016/j.ajhg.2018.11.002

Moyer, C. L., Ivanovich, J., Gillespie, J. L., Doberstein, R., Radke, M. R., Richardson, M. E., et al. (2020). Rare BRIP1 missense alleles confer risk for ovarian and breast cancer. Cancer Res. 80, 857-867. doi: 10.1158/0008-5472.Can-19- 1991

Narayan, G., Bourdon, V., Chaganti, S., Arias-Pulido, H., Nandula, S. V., Rao, P. H., et al. (2007). Gene dosage alterations revealed by cDNA microarray analysis in cervical cancer: identification of candidate amplified and overexpressed genes. Genes Chromosomes Cancer 46, 373-384. doi: 10.1002/gcc.20418

Nickoloff, J. A., Jones, D., Lee, S. H., Williamson, E. A., and Hromas, R. (2017). Drugging the cancers addicted to DNA repair. J. Natl. Cancer Inst. 109:djx059. doi: $10.1093 /$ jnci/djx059

Pashayan, N., Antoniou, A. C., Ivanus, U., Esserman, L. J., Easton, D. F., French, D., et al. (2020). Personalized early detection and prevention of breast cancer: ENVISION consensus statement. Nat. Rev. Clin. Oncol. 17, 687-705. doi: 10. 1038/s41571-020-0388-9

Pilié, P. G., Tang, C., Mills, G. B., and Yap, T. A. (2019). State-of-the-art strategies for targeting the DNA damage response in cancer. Nat. Rev. Clin. Oncol. 16, 81-104. doi: 10.1038/s41571-018-0114-z

Pradhan, A., Singh, T. R., Ali, A. M., Wahengbam, K., and Meetei, A. R. (2013). Monopolar spindle 1 (MPS1) protein-dependent phosphorylation of RecQmediated genome instability protein 2 (RMI2) at serine 112 is essential for BLM-Topo III $\alpha$-RMI1-RMI2 (BTR) protein complex function upon spindle assembly checkpoint (SAC) activation during mitosis. J. Biol. Chem. 288, 33500-33508. doi: 10.1074/jbc.M113.470823

Qin, G., Wang, X., Ye, S., Li, Y., Chen, M., Wang, S., et al. (2020). NPM1 upregulates the transcription of PD-L1 and suppresses $\mathrm{T}$ cell activity in triple-negative breast cancer. Nat. Commun. 11:1669. doi: 10.1038/s41467-02015364-Z

Quiros, S., Roos, W. P., and Kaina, B. (2011). Rad51 and BRCA2-New molecular targets for sensitizing glioma cells to alkylating anticancer drugs. PLoS One 6:e27183. doi: 10.1371/journal.pone.0027183 
Quist, J., Mirza, H., Cheang, M. C. U., Telli, M. L., O'Shaughnessy, J. A., Lord, C. J., et al. (2019). A Four-gene decision tree signature classification of triplenegative breast cancer: implications for targeted therapeutics. Mol. Cancer Ther. 18, 204-212. doi: 10.1158/1535-7163.Mct-18-0243

Rizeq, B., Sif, S., Nasrallah, G. K., and Ouhtit, A. (2020). Novel role of BRCA1 interacting C-terminal helicase 1 (BRIP1) in breast tumour cell invasion. J. Cell Mol. Med. 24, 11477-11488. doi: 10.1111/jcmm.15761

Roos, W. P., Thomas, A. D., and Kaina, B. (2016). DNA damage and the balance between survival and death in cancer biology. Nat. Rev. Cancer 16, 20-33. doi: $10.1038 /$ nrc. 2015.2

Rutter, J. L., Smith, A. M., Dávila, M. R., Sigurdson, A. J., Giusti, R. M., Pineda, M. A., et al. (2003). Mutational analysis of the BRCA1-interacting genes ZNF350/ZBRK1 and BRIP1/BACH1 among BRCA1 and BRCA2-negative probands from breast-ovarian cancer families and among early-onset breast cancer cases and reference individuals. Hum. Mutat. 22, 121-128. doi: 10.1002/ humu. 10238

San Filippo, J., Sung, P., and Klein, H. (2008). Mechanism of eukaryotic homologous recombination. Annu. Rev. Biochem. 77, 229-257. doi: 10.1146/ annurev.biochem.77.061306.125255

Scarbrough, P. M., Weber, R. P., Iversen, E. S., Brhane, Y., Amos, C. I., Kraft, P., et al. (2016). A cross-cancer genetic association analysis of the DNA repair and DNA damage signaling pathways for lung, ovary, prostate, breast, and colorectal cancer. Cancer Epidemiol. Biomarkers. Prev. 25, 193-200. doi: 10.1158/10559965.Epi-15-0649

Slebos, R. J., Yi, Y., Ely, K., Carter, J., Evjen, A., Zhang, X., et al. (2006). Gene expression differences associated with human papillomavirus status in head and neck squamous cell carcinoma. Clin. Cancer Res. 12(3 Pt 1), 701-709. doi: 10.1158/1078-0432.Ccr-05-2017

Sung, H., Ferlay, J., Siegel, R. L., Laversanne, M., Soerjomataram, I., Jemal, A., et al. (2021). Global Cancer Statistics 2020: GLOBOCAN Estimates of Incidence and Mortality Worldwide for 36 Cancers in 185 Countries. CA Cancer J. Clin. 71, 209-249. doi: 10.3322/caac. 21660

Teo, M. Y., Seier, K., Ostrovnaya, I., Regazzi, A. M., Kania, B. E., Moran, M. M., et al. (2018). Alterations in DNA damage response and repair genes as potential marker of clinical benefit from PD-1/PD-L1 blockade in advanced urothelial cancers. J. Clin. Oncol. 36, 1685-1694. doi: 10.1200/jco.2017.75.7740

Thul, P. J., Åkesson, L., Wiking, M., Mahdessian, D., Geladaki, A., Ait Blal, H., et al. (2017). A subcellular map of the human proteome. Science 356:eaal3321. doi: $10.1126 /$ science.aal3321

Tian, W., Shan, B., Zhang, Y., Ren, Y., Liang, S., Zhao, J., et al. (2020). Association between DNA damage repair gene somatic mutations and immune-related gene expression in ovarian cancer. Cancer Med. 9, 2190-2200. doi: 10.1002/cam4. 2849

Tsai, M. S., Kuo, Y. H., Chiu, Y. F., Su, Y. C., and Lin, Y. W. (2010). Downregulation of Rad51 expression overcomes drug resistance to gemcitabine in human non-small-cell lung cancer cells. J. Pharmacol. Exp. Ther. 335, 830-840. doi: 10.1124/jpet.110.173146

Turner, N., Tutt, A., and Ashworth, A. (2004). Hallmarks of 'BRCAness' in sporadic cancers. Nat. Rev. Cancer 4, 814-819. doi: 10.1038/nrc1457

Tutt, A., Tovey, H., Cheang, M. C. U., Kernaghan, S., Kilburn, L., Gazinska, P., et al. (2018). Carboplatin in BRCA1/2-mutated and triple-negative breast cancer BRCAness subgroups: the TNT Trial. Nat. Med. 24, 628-637. doi: 10. 1038/s41591-018-0009-7

Uhlén, M., Fagerberg, L., Hallström, B. M., Lindskog, C., Oksvold, P., Mardinoglu, A., et al. (2015). Proteomics. Tissue-based map of the human proteome. Science 347:1260419. doi: 10.1126/science.1260419

Uhlen, M., Zhang, C., Lee, S., Sjöstedt, E., Fagerberg, L., Bidkhori, G., et al. (2017). A pathology atlas of the human cancer transcriptome. Science 357:eaan2507. doi: 10.1126/science.aan 2507

Weber-Lassalle, N., Hauke, J., Ramser, J., Richters, L., Groß, E., Blümcke, B., et al. (2018). BRIP1 loss-of-function mutations confer high risk for familial ovarian cancer, but not familial breast cancer. Breast Cancer Res. 20:7. doi: 10.1186/ s13058-018-0935-9

Wengner, A. M., Scholz, A., and Haendler, B. (2020). Targeting DNA damage response in prostate and breast cancer. Int. J. Mol. Sci. 21:8273. doi: 10.3390/ ijms21218273

Xu, M. D., Liu, S. L., Zheng, B. B., Wu, J., Wu, M. Y., Zhang, Y., et al. (2018). The radiotherapy-sensitization effect of cantharidin: mechanisms involving cell cycle regulation, enhanced DNA damage, and inhibited DNA damage repair. Pancreatology 18, 822-832. doi: 10.1016/j.pan.2018.08. 007

Yao, N. Y., and O'Donnell, M. (2012). The RFC clamp loader: structure and function. Subcell. Biochem. 62, 259-279. doi: 10.1007/978-94-007-45 72-8_14

Yap, T. A., Plummer, R., Azad, N. S., and Helleday, T. (2019). The DNA damaging revolution: PARP inhibitors and beyond. Am. Soc. Clin. Oncol. Educ. Book 39, 185-195. doi: 10.1200/edbk_238473

Zhan, W., Liu, Y., Gao, Y., Gong, R., Wang, W., Zhang, R., et al. (2020). RMI2 plays crucial roles in growth and metastasis of lung cancer. Signal Transduct. Target Ther. 5:188. doi: 10.1038/s41392-020-00295-4

Zhang, W., Liu, B., Wu, W., Li, L., Broom, B. M., Basourakos, S. P., et al. (2018). Targeting the MYCN-PARP-DNA damage response pathway in neuroendocrine prostate cancer. Clin. Cancer Res. 24, 696-707. doi: 10.1158/1078-0432.Ccr-171872

Conflict of Interest: The authors declare that the research was conducted in the absence of any commercial or financial relationships that could be construed as a potential conflict of interest.

Publisher's Note: All claims expressed in this article are solely those of the authors and do not necessarily represent those of their affiliated organizations, or those of the publisher, the editors and the reviewers. Any product that may be evaluated in this article, or claim that may be made by its manufacturer, is not guaranteed or endorsed by the publisher.

Copyright (c) 2021 Wang, Gu, Chi, Liu, Xiong, Zhong, Wang, Wang and Li. This is an open-access article distributed under the terms of the Creative Commons Attribution License (CC BY). The use, distribution or reproduction in other forums is permitted, provided the original author(s) and the copyright owner(s) are credited and that the original publication in this journal is cited, in accordance with accepted academic practice. No use, distribution or reproduction is permitted which does not comply with these terms. 